\title{
Prevenção contra a COVID-19 em escolas do município de Parnaíba-PI: um relato de experiência sobre educação em saúde
}

\author{
Prevention against COVID-19 in schools of the municipality of Parnaíba-PI: an experience \\ report about health education
}

\author{
Prevención contra COVID-19 en escuelas del município de Parnaíba-PI: informe de \\ experiencia sobre educación para la salud
}

Martha Laura Leão dos Santos Silva ${ }^{1 *}$, Bruna Maria de Carvalho Pereira ${ }^{1}$, Sarah Nilkece Mesquita Araújo Nogueira Bastos ${ }^{1}$, Livia Rocha Santos ${ }^{1}$, Julliana Emily Matos e Silva ${ }^{1}$, Francisco Nogueira do Rego Neto ${ }^{1}$, Roberto Augusto Lopes Cajubá de Britto ${ }^{1}$, Luís Eduardo Soares Macêdo Mendes ${ }^{1}$, Vinicius Souza Freires ${ }^{1}$, Juliana Félix de Melo.

\section{RESUMO}

Objetivo: Relatar a experiência de uma ação educativa remota sobre prevenção da COVID-19 no município de Parnaíba-PI. Relato de experiência: Trata-se de um estudo descritivo do tipo relato de experiência abordando a experiência de nove discentes do curso de Medicina de uma universidade pública do Nordeste do Brasil. O grupo desenvolveu estratégias educativas em saúde acerca da prevenção da COVID-19 e avaliou o efeito da implementação destas estratégias. O público-alvo das ações constituiu-se em estudantes do $1^{\circ}$ ao 9 ano do ensino fundamental, de 11 escolas públicas e privadas. Desenvolveram-se estratégias educativas remotas como vídeos, músicas, teatro de marionetes e jogos acerca de ações preventivas da COVID-19. Participaram da intervenção 359 alunos. Além disso, foram aplicados dois formulários para avaliar o conhecimento dos alunos sobre o tema antes e depois da intervenção educativa. Considerações finais: A ação possibilitou a disseminação de medidas preventivas em relação à pandemia de COVID-19 com o intuito de mitigar a transmissão nos ambientes escolares. Também proporcionou uma evolução significativa no conhecimento dos alunos participantes com melhoria das habilidades para criação de atividades lúdicas no novo contexto de forma remota.

Palavras-chave: COVID-19, SARS-CoV-2, Ensino fundamental.

\begin{abstract}
Objective: Communicate an experience of remote educational action on the prevention of COVID-19 in the city of Parnaíba-PI. Experience report: This is a descriptive study of the experience report type, approaching the experience of nine students of the Medicine course of a University in the Northeast of Brazil. The group developed health education strategies about the prevention of COVID-19 and evaluated the effect of implementing these strategies. The target audience of the actions consisted of students from the 1 st to the 9th grade of elementary school, from 11 public and private schools. Remote educational strategies were developed such as videos, music, puppet theater and games about preventive actions of COVID-19. 359 students participated in the intervention. In addition, there were two forms to assess students' knowledge on the topic before and after the educational intervention. Final considerations: The action enabled the dissemination of preventive measures in relation to the COVID-19 pandemic in order to mitigate transmission in school environments. It also provided a need for knowledge of participating students with the improvement of skills to create playful activities in the new context remotely.
\end{abstract}

Key words: COVID-19, SARS-CoV-2, Primary education.

${ }^{1}$ Universidade Federal do Delta do Parnaíba (UFDPar), Parnaíba - PI.

*E-mail: marthalauraleaodosssilva@gmail.com 


\section{RESUMEN}

Objetivo: Informar la experiencia de una acción educativa a distancia sobre la prevención de COVID-19 en la ciudad de Parnaíba-PI. Informe de experiencia: Se trata de un estudio descriptivo del tipo informe de experiencia, que aborda la experiencia de nueve estudiantes de la carrera de Medicina de una Universidad pública en el Nordeste de Brasil. El grupo desarrolló estrategias de educación para la salud sobre la prevención de COVID-19 y evaluó el efecto de implementar estas estrategias. El público objetivo de las acciones fue el alumnado de $1^{\circ}$ a $9^{\circ}$ de primaria, de 11 colegios públicos y privados. Se desarrollaron estrategias educativas a distancia, como videos, música, teatro de marionetas y juegos sobre acciones preventivas del COVID-19. 359 estudiantes participaron en la intervención. Además, se aplicaron dos formularios para evaluar el conocimiento de los estudiantes sobre el tema antes y después de la intervención educativa. Consideraciones finales: La acción permitió la difusión de medidas preventivas en relación a la pandemia COVID-19 con el fin de mitigar la transmisión en ambientes escolares. También proporcionó una importante evolución en el conocimiento de los estudiantes participantes, mejorando sus habilidades para crear actividades lúdicas de forma remota en el nuevo contexto.

Palabras clave: COVID-19, SARS-CoV-2, Educación primaria.

\section{INTRODUÇÃO}

Com a chegada da pandemia de COVID-19 no Brasil, muitas medidas de prevenção foram adotadas em todas as regiões do país. No estado do Piauí o novo coronavírus, denominado SARS-CoV-2, se alastrou rapidamente a partir de março de $2020 \mathrm{com}$ os primeiros casos na capital Teresina e em pouco tempo várias cidades foram atingidas. Escolas e universidades fecharam as portas temporariamente para 0 ensino presencial e muitos desafios surgiram na área de educação para o enfrentamento da pandemia (OLIVEIRA ES, et al., 2020).

De acordo com a Organização das Nações Unidas para a Educação, a Ciência e a Cultura (UNESCO), $91 \%$ da população estudantil mundial foi afetada com esse fechamento (UNESCO, 2020). As comunidades mais vulneráveis foram também mais afetadas pelo surto por não poderem continuar com a aprendizagem à distância (TUFAN ZK e KAYAASLAN B, 2020).

Como não há ainda um tratamento eficaz contra a COVID-19, a melhor opção para o controle da doença é a vacinação (SHIH HI, et al., 2020). No entanto, até que a cobertura vacinal seja completa e a transmissão do vírus controlada, a população necessita adotar medidas para se proteger da doença. Sendo assim, as escolas precisam adotar ações de controle da infecção ao retornarem suas atividades de forma presencial (DIAS GN, et al., 2020).

No ambiente escolar, a disseminação de infecções torna-se maior tanto pela proximidade entre os alunos quanto pelo fato das aulas ocorrerem em locais fechados. Além disso, atos que eram comuns antes da pandemia de COVID-19 tais como compartilhamento de objetos pessoais potencializam o risco de disseminação da doença (CELESTINO JÚNIOR AF, et al., 2017). Nesse contexto, cuidados simples como a higienização correta das mãos e o incentivo às ações de prevenção auxiliam na redução dos danos (SOUZA ES, et al., 2015).

A partir do início de 2020, o Ministério da Saúde (MS) tem orientado a população quanto à importância de medidas de prevenção contra o SARS-CoV-2. A lavagem correta das mãos, o distanciamento social e a utilização de locais abertos e ventilados fazem parte destas medidas recomendadas. Em abril de 2020, além das ações citadas, houve também recomendação quanto ao uso de máscaras para mitigar a propagação do vírus (BRASIL, 2020a; OLIVEIRA WK, et al., 2020).

Dados da Pesquisa Nacional de Saúde do Escolar (PeNSE) de 2015 demonstram a necessidade de modificação na rotina de higiene de estudantes brasileiros do ensino fundamental. Para tal conclusão foram utilizados indicadores que avaliaram a lavagem de mãos antes de realizar as refeições e após a utilização 
dos banheiros. O resultado indicou uma pequena adesão dos estudantes às técnicas corretas de higienização das mãos (INSTITUTO BRASILEIRO DE GEOGRAFIA E ESTATÍSTICA, 2016).

Atualmente, várias medidas são necessárias para o controle da COVID-19, sendo as instituições escolares ambientes importantes para a realização de práticas de educação em saúde após a volta às aulas (ORGANIZAÇÃO PAN-AMERICANA DA SAÚDE, 2020).

Os alunos precisam de informações corretas sobre a pandemia de COVID-19 para se protegerem e diminuírem a transmissão do vírus na comunidade. Sendo assim, este estudo objetivou descrever ações realizadas por um grupo de estudantes de medicina com o intuito de disseminar informações acerca da prevenção da COVID-19 entre alunos de instituições escolares do $1^{\circ}$ ao $9^{\circ}$ anos do ensino fundamental do município de Parnaíba-PI.

\section{RELATO DE EXPERIÊNCIA}

Este é um estudo descritivo que aborda a experiência de nove discentes do curso de Medicina participantes do projeto intitulado "COVID-19: ações preventivas entre crianças e adolescentes do ensino fundamental do município de Parnaíba-PI". Este projeto foi criado no ano de $2020 \mathrm{com}$ o intuito de desenvolver atividades entre estudantes do ensino fundamental. O grupo desenvolveu estratégias educativas em saúde acerca da prevenção da COVID-19 e avaliou o efeito da implementação destas estratégias nas escolas que participaram.

Respeitando as normas de distanciamento social, as atividades foram realizadas por meio de plataformas virtuais. O público-alvo das ações constituiu-se em estudantes do $1^{\circ}$ ao $9^{\circ}$ ano do ensino fundamental, de 11 escolas públicas e privadas. Foram realizadas reuniões semanais para o planejamento das ações e para 0 melhor desenvolvimento destas foi decidido dividir os 9 acadêmicos em 3 grupos com 3 pessoas devido à faixa etária diversificada que exigiu diferentes metodologias para 0 aprendizado eficaz das medidas preventivas. Assim, o grupo 1 desenvolveu atividades para crianças do $1^{\circ}$ ao $3^{\circ}$ ano, o grupo 2 para alunos do $4^{\circ}$ ao $6^{\circ}$ ano e o grupo 3 para os participantes do $7^{\circ}$ ao $9^{\circ}$ ano do ensino fundamental.

Em cada um dos três grupos, após a construção dos materiais foram criados dois formulários com o intuito de avaliar o conhecimento dos alunos sobre o tema. Os formulários foram estruturados para serem aplicados da seguinte forma: o primeiro deveria ser respondido antes dos estudantes terem acesso aos conteúdos criados, para avaliar o conhecimento prévio dos alunos e o segundo formulário seria respondido após as atividades para verificar o aprendizado e fixação de novas informações assim como o conhecimento depois da intervenção educativa.

A abordagem das crianças com menor faixa etária demandou a produção de ações mais lúdicas que fixassem a atenção. Assim, optou-se pela elaboração de um vídeo educativo utilizando marionetes, outro sobre os conceitos principais da COVID-19 e uma paródia. No primeiro vídeo, denominado "Uma consulta legal", optou-se por trazer duas marionetes, representando uma médica e uma criança, que apresentava dúvidas referentes à pandemia para a profissional e esta dava orientações sobre os principais cuidados de prevenção contra o SARS-CoV-2. No vídeo, erros foram inseridos intencionalmente, para que as crianças detectassem as falhas, como a ausência de máscaras das personagens e falta de distanciamento mínimo entre as mesmas.

O segundo vídeo foi em formato de aula com tela interativa na qual uma professora explica para as crianças informações gerais sobre a COVID-19. O terceiro vídeo foi uma paródia, com formato dinâmico e diversas imagens e desenhos interativos que demonstrou as etiquetas e comportamentos higiênicos na pandemia.

Das atividades do grupo 1 , do $1^{\circ}$ ao $3^{\circ}$ ano participaram 115 estudantes, no entanto, incluíram-se na amostra apenas 91, que completaram integralmente os formulários de avaliação antes e após a intervenção. Destes, a maior adesão foi entre alunos do $3^{\circ}$ ano. A média de acertos das respostas dos alunos acerca das perguntas sobre prevenção da Covid-19 foi menor durante o pré-teste, comparada a maior quantidade de 
acertos durante o pós-teste. Todos possuíam idade entre 6 e 9 anos. Sobre o apontamento das falhas intencionais no vídeo com marionetes, a maioria dos estudantes reconheceram pelo menos uma falha no vídeo. Em relação à satisfação com a intervenção, todos os estudantes consideraram a atividade prazerosa e de fácil compreensão.

A abordagem das crianças do $4^{\circ}$ ao $6^{\circ}$ ano demandou a produção de ações que estimulassem a participação dos alunos e prendessem sua atenção. Portanto, decidiu-se elaborar um jogo de perguntas e respostas sobre os principais pontos relacionados à COVID-19, como transmissão e prevenção. O jogo, intitulado "COVID GAME", possui cinco questões de múltipla escolha com quatro alternativas com apenas uma correta. Após cada questão, iniciava-se uma explicação sobre o gabarito, com o objetivo de compartilhar informações sobre o tema abordado.

Além disso, optou-se pela criação de um panfleto com informações essenciais sobre a Covid-19 e um vídeo com curiosidades sobre os coronavírus. O vídeo, " 5 curiosidades: coronavírus", aborda de forma rápida curiosidades como o local onde surgiram os primeiros casos, diferentes nomenclaturas e outros tópicos. A gravação tem duração de 5 minutos e foi editada e roteirizada pelos alunos do grupo 2 .

Do grupo 2 participaram 153 estudantes, no entanto, incluíram-se na amostra apenas 108, pois completaram integralmente os formulários de conhecimento prévio e verificação de aprendizagem após o desenvolvimento das atividades. $\mathrm{Na}$ análise das respostas, foi obtida maior adesão entre alunos do $5^{\circ}$ ano. Quanto às idades, todos possuíam pelo menos 9 anos. A média de acertos foi menor durante o pré-teste, aumentando durante pós-teste, levando em consideração 1 ponto para cada uma das 5 questões em que houve acerto. Quando perguntados sobre a satisfação com a intervenção, os estudantes e os professores consideraram a atividade fácil e rápida.

A abordagem dos alunos do $7^{\circ}$ ao $9^{\circ}$ ano permitiu um enfoque mais técnico com um maior número de informações, tendo em vista uma maior faixa etária e maior nível de escolaridade. A abordagem foi feita em um site criado pelos membros do grupo através da plataforma Google Sites a fim de reunir e facilitar o acesso a todo conteúdo produzido.

Dois vídeos de curta duração foram produzidos elucidando as temáticas citadas anteriormente, intitulados "Técnicas de lavagem correta das mãos" e "Informações iniciais e o uso correto da máscara". Para a produção de ambos, fez-se uso do roteiro criado pelos integrantes do grupo. Os curtas possuem imagens ilustrativas de domínio público com narração e explicação das mesmas pelos membros. Para complementar os vídeos, informações em forma de textos e imagens também foram adicionadas. Do grupo 3, houve um total de 160 alunos que aderiram à atividade e, posteriormente, completaram a atividade solicitada. Considerando a participação de alunos do $7^{\circ}, 8^{\circ}$ e $9^{\circ}$ ano, foi obtida maior adesão entre os alunos do $9^{\circ}$ ano. Quanto às idades, variaram entre 12 e acima de 14 anos. Também vale destacar que a média de acertos evoluiu do pré-teste para o pós-teste, o que reflete maiores acertos após a aplicação dos vídeos.

\section{DISCUSSÃO}

A execução deste trabalho possibilitou a disseminação de medidas preventivas em meio a pandemia de COVID-19 com o intuito de mitigar a transmissão nos ambientes escolares. Além disso, os participantes do projeto melhoraram habilidades relacionadas à educação em saúde por meio de plataformas e ferramentas virtuais.

Recomendações que incentivaram o distanciamento social iniciaram-se em Wuhan na China e pouco tempo depois vários outros países começaram a tomar medidas semelhantes (SILVA LLS, et al., 2020). No Brasil, o Ministério da Educação interrompeu as aulas presenciais e orientou a substituição destas por meios digitais enquanto durasse a pandemia do novo coronavírus (BRASIL, 2020b). Nesse cenário, o presente projeto atuou para informar sobre medidas necessárias com o retorno das aulas de maneira presencial.

Infecções respiratórias como a COVID-19 se espalham de forma rápida em ambientes fechados como as escolas. Informações sobre métodos preventivos destas doenças contribuem significativamente para a 
redução das mesmas, sendo a escola um ambiente propício para trabalhar o tema. Uma destas informações é a orientação quanto à forma correta de lavagem de mãos. Ações que visam disseminar tais dados contribuem para a redução da transmissão e evitam surtos que podem afetar toda a escola (CELESTINO JÚNIOR AF, et al., 2017). Neste âmbito, estudo realizado por Willmott M, et al. (2015) mostrou o impacto na redução da transmissão de doenças nas escolas com essas atividades.

Os participantes do projeto atuaram de forma remota, por meio de formulários online. Nesse sentido, é importante ressaltar que um dos maiores desafios para o ensino a distância é a desigualdade econômica. As aulas ministradas por meios digitais requerem dispositivos e aplicativos, o que dificulta a chegada do conteúdo e diminui a integração de alunos mais carentes. Parte da população possui em casa ambiente propício para estudo e dispositivos eletrônicos individuais com internet rápida, enquanto outra parte não possui este acesso (OLIVEIRA ES, et al., 2020). Assim, no Brasil, a desigual distribuição de renda diminui a inclusão digital e cria entraves para a democratização do uso de tecnologias (ALMEIDA CCR, et al., 2019).

Outrossim, as atividades desenvolvidas pelo projeto incluíram uma faixa etária bastante variável. Sabe-se que as intervenções que visam promover a saúde nas escolas muitas vezes não atingem o objetivo por não abordarem temas de relevância para a faixa etária do público-alvo ou mesmo por não trabalharem os temas de forma que desperte o interesse deste (PALACIO DQA, et al., 2021). No entanto, quando há um estudo prévio do grupo o qual a atividade se destina tal estratégia torna-se um instrumento eficiente de repasse de informações verdadeiras e que auxiliam na prevenção de agravos à saúde (BUBADUÉ RM, et al., 2020). A utilização de metodologias ativas no processo de educação em saúde auxilia bastante na fixação do conhecimento e transcende as aulas puramente expositivas nas quais os alunos, na maioria das vezes, não participam ativamente (ASSUNÇÃO A, 2021).

No contexto de aulas remotas, muitos alunos foram prejudicados por não possuírem acesso à internet $\mathrm{e}$ aos dispositivos móveis. As comunidades mais vulneráveis foram também mais afetadas pelo surto por não poderem continuar com a aprendizagem remota ou à distância (TUFAN ZK e KAYAASLAN B, 2020). Desse modo, as aulas presenciais só podem retornar à medida que a situação epidemiológica se tornar favorável. Para que isso seja feito de forma segura, ações como a medição da temperatura, a utilização de máscaras e a troca a cada duas horas, o número de alunos reduzido e a disponibilização de álcool em gel e pia com sabão para lavagem das mãos são fundamentais (DIAS GN, et al., 2020). Ademais, é necessário o treinamento dos profissionais que trabalham no ambiente escolar para identificação de sintomas e uso correto dos Equipamentos de Proteção Individual (MINISTÉRIO DA SAÚDE, 2020).

Durante a construção do projeto foi possível aprimorar habilidades com metodologias ativas e lúdicas. A experiência com o desenvolvimento de softwares educativos, sites, vídeos e jogos interativos foi essencial para que os discentes entendessem o processo de ensino-aprendizagem como algo dinâmico e que extrapola para a educação em saúde e para a vida profissional futura. No tocante à pesquisa e busca em bases de dados, para organizar o arcabouço teórico sobre o conteúdo, foi necessária uma extensa atualização nas melhores evidências sobre a temática da COVID-19 e sobre os aspectos inerentes ao SARS-CoV-2. Isso porque um dos desafios encontrados atualmente é a disseminação de informações falsas acerca da doença (NETO M, et al., 2020).

As atividades desenvolvidas pelo grupo disseminaram conhecimento e cumpriram o papel de informar acerca da prevenção contra a COVID-19. Apesar dos desafios encontrados tais como o difícil acesso à internet por alguns alunos, o uso de metodologias à distância e a necessidade das crianças menores de terem o acompanhamento dos pais para responder, a maior parte dos alunos avaliou o projeto como produtivo e de fácil compreensão. Sugere-se que mais estudos sejam realizados neste contexto para que outras doenças infecciosas possam ser evitadas no ambiente escolar.

\section{REFERÊNCIAS}

1. ALMEIDA CCR, et al. Inserção digital e desigualdades na demanda por cultura no Brasil. Nova Economia, 2019; 29: 1221-1247.

REAS | Vol. 15 (1) | DOI: https://doi.org/10.25248/REAS.e9532.2022 
2. ASSUNÇÃO A. Metodologias ativas de aprendizagem: práticas no ensino da Saúde Coletivas para alunos de Medicina. Revista Brasileira de Educação Médica, 2021; 45(3).

3. BRASIL. Máscaras caseiras podem ajudar na prevenção contra o coronavírus. 2020a. Disponível em: https://www.saude.gov.br/noticias/ agencia-saude/46645-mascaras-caseiras-podemajudar-na-prevencao-contra-ocoronavirus. Acessado em: 6 de setembro de 2021.

4. BRASIL. Portaria № 343, de 17 de março de 2020. Dispõe sobre a substituição das aulas presenciais por aulas em meios digitais enquanto durar a situação de pandemia do Novo Coronavírus. 2020b. Disponível em: https://www.in.gov.br/en/web/dou/-/Portaria-n-343-de-17-de-marco-de-2020-248564376. Acessado em: 14 de setembro de 2021.

5. BUBADUÉ RM, et al. Oficinas de educação em saúde com crianças no contexto da pandemia COVID-19. Revista Brasileira de Enfermagem, 2020; 73(2).

6. CELESTINO JÚNIOR AF, et al. Riscos infecciosos no ambiente escolar: relato de experiência com escolares através de metodologia ativa. Saúde \& Transformação Social, 2017; 8(2): 128-134.

7. DIAS GN, et al. Retorno às aulas presenciais no sistema educacional do estado do Pará-Brasil: Obstáculos e desafios durante a epidemia de Covid-19 (Sars-Cov-2). Brazilian Journal of Development, 2020; 6(6): 37906-37924.

8. INSTITUTO BRASILEIRO DE GEOGRAFIA E ESTATísTICA. Ministério do Planejamento, Orçamento e Gestão. Diretoria de Pesquisas. Coordenação de População e Indicadores Sociais. Pesquisa Nacional de Saúde Escolar 2015. 2016. Disponível em: https://biblioteca.ibge.gov.br/visualizacao/livros/liv97870.pdf. Acessado em: 6 de setembro de 2021.

9. MINISTÉRIO DA SAÚDE. Contribuições para o retorno às atividades escolares presenciais no contexto da pandemia Covid-19. $2020 . \quad$ Disponível em: https://www.arca.fiocruz.br/bitstream/icict/43379/2/contribuicoes_para_o_retorno_escolar_-_08.09_4_1.pdf. Acessado em: 2 de novembro de 2021.

10. NETO M, et al. Fake news no cenário da pandemia de COVID-19. Cogitare enfermagem, 2020; 25.

11. OLIVEIRA ES, et al. A educação a distância $(E a D)$ e os novos caminhos da educação após a pandemia ocasionada pela Covid-19. Brazilian Journal of Development, 2020; 6(7): 52860-52867.

12. OLIVEIRA WK, et al. Como o Brasil pode deter a COVID-19. Epidemiologia e Serviços de Saúde, 2020; 29(2): e2020044.

13. ORGANIZAÇÃO DAS NAÇÕES UNIDAS PARA A EDUCAÇÃO, A CIÊNCIA E A CULTURA (UNESCO). COVID-19 Educational Disruption and Response. 2020. Disponível em: https://en.unesco.org/covid19/educationresponse. Acessado em: 6 de setembro de 2021.

14. ORGANIZAÇÃO PAN-AMERICANA DA SAÚDE. Mensagens e ações importantes para a COVID-19 prevenção e controle em escolas. 2020. Disponível em: https://doi.org/OPAS/BRA/Covid-19/20-015. Acessado em: 6 de setembro de 2021.

15. PALACIO DQA, et al. Aspectos relacionados à saúde do jovem escolar: uma revisão. Revista Eletrônica Acervo Saúde, 2021; 13(4): e6964.

16. SHIH HI, et al. Fighting COVID-19: A quick review of diagnoses, therapies, and vacines. Biomedical Journal, 2020; 43(4): 341-354.

17. SILVA LLS, et al. Medidas de distanciamento social para o enfrentamento da COVID-19 no Brasil: caracterização e análise epidemiológica por estado. Cadernos de Saúde Pública, 2020; 36(9).

18. SOUZA ES, et al. Mortalidade e riscos associados a infecção relacionada à assistência à saúde. Texto \& Contexto Enfermagem [online], 2015; 24(1): 220-228.

19. TUFAN ZK, KAYAASLAN B. Crushing the curve, the role of national and international institutions and policy makers in COVID-19 pandemic. Turkish Journal of Medical Sciences, 2020; 50(3): 495-508.

20. WILLMOTT $M$, et al. Effectiveness of hand hygiene interventions in reducing illness absence among children in educational settings: a systematic review and meta-analysis. Archives of Disease in Childhood, 2015; 101(1): 42-50. 